\title{
Inclusion of Classic HVDC links in a PMU-Based State Estimator
}

\author{
W. Li, Student Member, IEEE, L. Vanfretti, Member, IEEE
}

\begin{abstract}
This article presents a PMU-based state estimator algorithm that considers the presence of classic HVDC links. A hybrid AC/DC network model is developed to combine the AC network and DC links. The algorithm assumes that DC link measurements can be sampled synchronously, are time-stamped and reported at the same rate as PMU measurements. Then, by applying the weighted least squares algorithm, a linear state estimator can solve for both $\mathrm{AC}$ and DC states simultaneously. To validate the algorithm, a state estimation model is built for a 9-bus and the KTH-Nordic 32 systems, for both AC-only and hybrid AC/DC networks. In addition, the influence of losing DC measurements and effects of measurement noise on the state estimator are briefly discussed.
\end{abstract}

Index Terms-State estimation, Classic HVDC link, PMU, Hybrid AC and DC state estimation

\section{INTRODUCTION}

Power system state estimation (SE) is a process used to determine the state of a network by using measurements and a model of the grid. With a proper measurement redundancy level, SE can eliminate the effect of bad data and produce reliable state estimates of the grid in order to help operators monitor the power system and to support other analysis functions in Energy Management Systems (EMS) [1]. However, as electrical power systems continue to be expanded into larger interconnected networks spanning vast geographical areas [2] and are being developed to accommodate significant amounts of intermittent generation sources through conventional AC and new DC transmission technologies [3]; conventional SE will face the following challenges: (i) proper modeling [4] to take into account the models of point-to-point and even meshed DC networks embedded within a large AC grid; and (ii) to provide fast and accurate estimates that are capable of tracking a system's dynamic performance, specially over widespread geographical regions [5], considering both AC and DC networks simultaneously.

Traditionally, conventional SE is performed using a combination of measurements and pseudo-measurements including bus voltage magnitudes, active power flow and injection, reactive power flow and injection, current flow, etc.; taken at an asynchronous and low-sampling rate (2-4 seconds) [1]. In contrast, PMUs offer time-synchronized voltage and current phasor measurements over widespread geographical regions, which may allow new SE methods to trace the trajectories of the power system's response high higher granularity [2] (i.e.

W. Li and L. Vanfretti are with the Electric Power Systems Department, KTH Royal Institute of Technology, Stockholm, Sweden. E-mail: wei3@kth.se,luigiv@kth.se.

L. Vanfretti is with Statnett SF, Research \& Development, Oslo, Norway. E-mail: luigi.vanfretti@statnett.no.

W. Li is supported by the Swedish Energy Agency, Svenska Kraftnät, and ABB. L. Vanfretti is supported by Statnett SF and the STandUP for Energy collaboration initiative. with time-synchonized measurements at a rate of 30, 50, 100 or 120 samples per second [6]).

As for the network model, conventional SE is performed on a bus/branch model similar to that used in power flow calculations [1]. These models contain a large amount of nonlinear relationships. Although conventional SEs including classic HVDC links have been developed [7][8], the use of conventional measurements makes these SEs complex. In contrast, PMU-only SEs [5], [9], [10] use Kirchhoff's laws with voltage and current phasors, which significantly reduce the nonlinearities in the model compared to the power flow network equations, as well as reducing the computation burden of the numerical solution algorithms. On the other hand, both of them may apply a similar optimization algorithm to obtain an SE solution, namely the weighted least squares method.

This article presents an SE algorithm that takes DC links into consideration so that a PMU-based SE for hybrid AC/DC networks becomes feasible. The algorithm uses PMU measurements from the AC network, and DC voltage and DC current measurements sampled synchronously, time-stamped by GPS and at the same rate as PMU data. The network model utilized builds on the work in [10] and extends it to include classic HVDC links.

The reminder of article is organized as follows: First, in Section II, the model of both AC and DC power system components and network interfaces are introduced, which will help to build up the network model for hybrid SE. Second, in Section III, Phadke's and Thorp's linear state estimator (PTLSE) is extended to include classic HVDC links. This LSE is validated through simulations in two different test systems in Section IV. Additionally, issues regarding DC measurement loss and the influence of measurement noises on the SE are addressed. Conclusions and future work are discussed in Section V.

\section{Hybrid AC/DC Network Model}

\section{A. AC Network}

Similarly to [5], [9], the AC network model is comprised of a line with series admittance and shunt admittance, as well as transformers. All these components are enough to formulate the AC network model, which describes the relation between complex voltages on buses and complex currents flowing through the lines connected to these buses. This approach is similar to [11][12], but taking the shunt admittances and transformers into consideration. As shown in Fig. 1, the subscript $\boldsymbol{f}$ denotes the bus where current flows from and $t$ is the bus where current flows to. The $i$-th line is represented by a series admittance $y_{i}$ and shunt admittance $y_{i 0}$ in per unit. An ideal transformer is represented by the off-nominal tap ratio $a: 1$. In the case of phase shifting transformers, $a$ is a complex number. Consider a fictitious bus $x$ between the 


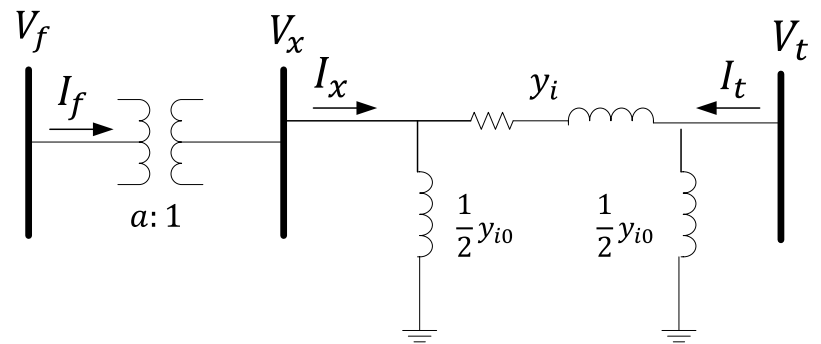

Fig. 1. An AC network model in consideration of transformer [11][12]

ideal transformer and the line series admittance, thus, for the assumed current directions, we have

$$
\tilde{V}_{x}=\frac{1}{a} \tilde{V}_{f}, \tilde{I}_{x}=a^{*} \tilde{I}_{f} .
$$

The current $\tilde{I}_{x}$ and $\tilde{I}_{t}$ are given by

$$
\tilde{I}_{x}=-y_{i} \tilde{V}_{t}+\left(y_{i}+\frac{1}{2} y_{i 0}\right) \tilde{V}_{x}, \tilde{I}_{t}=\left(y_{i}+\frac{1}{2} y_{i 0}\right) \tilde{V}_{t}-y_{i} \tilde{V}_{x} .
$$

Substituting for $\tilde{I}_{x}$ and $\tilde{V}_{x}$, we have

$$
\begin{aligned}
a^{*} \tilde{I}_{f} & =-y_{i} \tilde{V}_{t}+\frac{1}{a}\left(y_{i}+\frac{1}{2} y_{i 0}\right) \tilde{V}_{f} \\
\tilde{I}_{t} & =\left(y_{i}+\frac{1}{2} y_{i 0}\right) \tilde{V}_{t}-\frac{1}{a} y_{i} \tilde{V}_{f} .
\end{aligned}
$$

Writing the above equations in matrix form results in

$$
\left[\begin{array}{c}
\tilde{I}_{f} \\
\tilde{I}_{t}
\end{array}\right]=\underbrace{\left[\begin{array}{cc}
\frac{y_{i}+\frac{1}{2} y_{i 0}}{a^{2}} & -\frac{y_{i}}{a^{*}} \\
-\frac{y_{i}}{a} & y_{i}+\frac{1}{2} y_{i 0}
\end{array}\right]}_{Y_{A C}}\left[\begin{array}{c}
\tilde{V}_{i} \\
\tilde{V}_{j}
\end{array}\right]
$$

\section{B. DC Link Model}

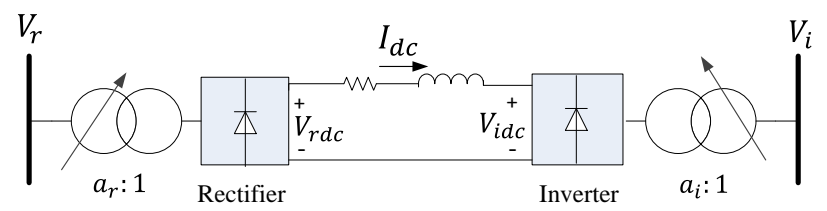

Fig. 2. A simplified DC link model [12][14]

A simplified DC link model is shown in Fig. 2. The subscript $\mathbf{r}$ refers to the rectifier side of the DC link and $\mathbf{i}$ refers to the inverter. For this classic HVDC link, the network equations are given by [12][13][14]:

$$
\begin{aligned}
& V_{r d c}=0.995 * \frac{3 \sqrt{2}}{\pi} a_{r}\left|\tilde{V}_{r}\right| \cos \alpha-\frac{3}{\pi} X_{r} I_{d c} \\
& V_{i d c}=0.995 * \frac{3 \sqrt{2}}{\pi} a_{i}\left|\tilde{V}_{i}\right| \cos \delta-\frac{3}{\pi} X_{i} I_{d c} \\
& I_{d c}=\frac{1}{R_{d c}}\left(V_{r d c}-V_{i d c}\right)
\end{aligned}
$$

where $a_{r}$ and $a_{i}$ are the tap ratios of the rectifier and inverter sides; $\alpha$ and $\delta$ are the firing angle and extinction angle; $X_{r}$ and $X_{i}$ are the transformer reactances of rectifier and inverter sides, respectively.

For hybrid $\mathrm{AC} / \mathrm{DC} \mathrm{SE}$, in addition to the $\mathrm{AC}$ bus voltage phasors, the rectifier side DC voltages, inverter side DC voltages, DC currents and products $\left|\tilde{V}_{r}\right| \cos \alpha$ and $\left|\tilde{V}_{i}\right| \cos \delta$ are also state variables. As shown in (2), the voltage magnitudes on the $\mathrm{AC}$ sides of the DC links may vary in time-especially when the system is subject to a disturbance. If we consider $\left|\tilde{V}_{r}\right|$ and $\left|\tilde{V}_{i}\right|$ as states, the DC network model will be nonlinear.

The proposed solution is to take $\left|\tilde{V}_{r}\right| \cos \alpha$ and $\left|\tilde{V}_{i}\right| \cos \delta$ as state variables of the DC link model. With this choice, a linear model of (2) is obtained as follows

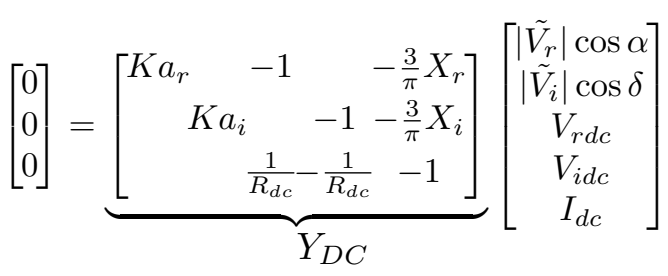

where $K=0.995 * \frac{3 \sqrt{2}}{\pi}$. Since $\tilde{V}_{r}$ and $\tilde{V}_{i}$ are state variables of AC network, $\tilde{V}_{r}$ and $V_{i}$ will be known after each SE snapshot. Then, $\cos \alpha$ and $\cos \delta$ can be derived.

\section{Hybrid AC/DC MeAsurement Model AND LineAR STATE ESTIMATION ALgORITHM}

\section{A. Linear State Estimation Algorithm}

Phadke's and Thorp's linear state estimator (PTLSE) [10] applies the Weighted Linear Least Squares (WLLS) method by using complex bus voltage and line current phasors in rectangular coordinates. In order to use WLLS, a linear function has to be defined first. In PTLSE, the state $\mathbf{x}$ is a vector of complex bus voltage phasors, and measurements $\mathrm{y}$ are both complex bus voltages and line currents phasors. Hence the linear function of the AC network is given by

$$
\left[\begin{array}{l}
\mathbf{V}_{\mathbf{m}} \\
\mathbf{I}_{\mathbf{m}}
\end{array}\right]=\left[\begin{array}{l}
\mathbf{U} \\
\mathbf{Y}
\end{array}\right] \mathbf{x}=\mathbf{A x}
$$

where the state vector $\mathbf{x}$ is comprised by complex voltage phasors, $\mathbf{U}$ is an identity matrix with rows corresponding to metered buses, and $\mathbf{Y}$ indicates the relation between line currents and bus voltages on both ends of the line. Substituting the network model into the measurement model, we obtain

$$
\left[\begin{array}{c}
\mathbf{r}_{\mathbf{V}} \\
\mathbf{r}_{\mathbf{I}}
\end{array}\right]=\left[\begin{array}{c}
\mathbf{U} \\
\mathbf{Y}
\end{array}\right] \mathbf{x}-\left[\begin{array}{l}
\mathbf{V}_{\mathbf{m}} \\
\mathbf{I}_{\mathbf{m}}
\end{array}\right]=\mathbf{A x}-\left[\begin{array}{c}
\mathbf{V}_{\mathbf{m}} \\
\mathbf{I}_{\mathbf{m}}
\end{array}\right]
$$

\section{B. Measurements Model and Linear State Estimation for AC Networks}

For the $\mathrm{AC} \mathrm{SE}$, a vector of complex voltage phasors is chosen as state variables while the measurements vector considers both current and voltage phasors. In order to obtain the relation between current and voltage for each line, namely $\mathbf{Y}$, the AC network model is used. Substituting (1) into (5), the linear relation between measurements and state vector of the $\mathrm{AC}$ network is given by

$$
\left[\begin{array}{c}
\mathbf{r}_{\mathbf{V}} \\
\mathbf{r}_{\mathbf{I}}
\end{array}\right]=\left[\begin{array}{cc}
1 & \\
\ddots & \\
- & 1 \\
\mathbf{Y}_{\mathbf{A C}}
\end{array}\right]\left[\begin{array}{c}
\tilde{V}_{1} \\
\vdots \\
\tilde{V}_{n}
\end{array}\right]-\left[\begin{array}{c}
\mathbf{V}_{\mathbf{m}} \\
\mathbf{I}_{\mathbf{m}}
\end{array}\right]
$$


C. Measurements Model and Linear State Estimation for hybrid AC/DC systems

For the hybrid AC/DC SE, the AC network model and DC link model are combined together. Thus, the linear relation between measurements and the state vector of a hybrid AC/DC system can be written as

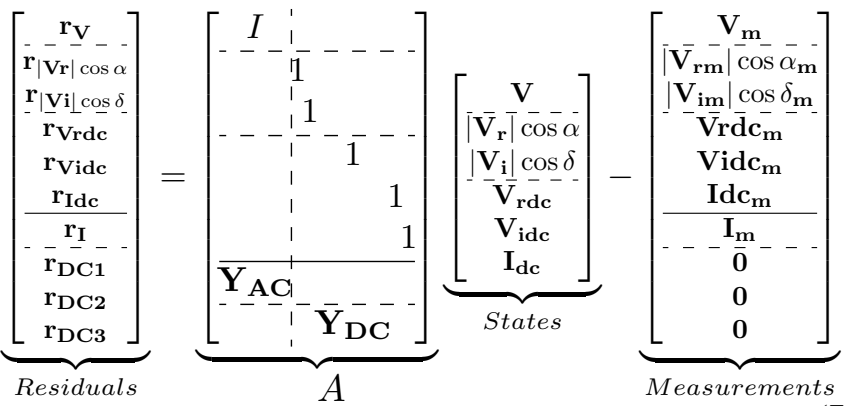

In the following sections, it is assumed that PMU measurements $\left(V_{r d c m}, V_{i d c m}, I_{d c m}, \cos \alpha_{m}\right.$ and $\left.\cos \delta_{m}\right)$ are available at all buses and that all DC measurements are available at the same reporting rate as the PMU data. Therefore, if all measurements can be obtained at each time instant (e.g. every $20 \mathrm{~ms}$ ), it is feasible to use a fast "static" SE to "recover" the dynamic changes of the system by obtaining an SE solution at each time instant.

\section{CAse Studies}

A 9-bus system [14] and the KTH-Nordic 32 system [15] are used to illustrate both AC-only and hybrid AC/DC SE results. Given space restrictions, the one-line diagrams of the original AC test systems will not be shown. The reader is referred to [14] for the 9-bus system and to [15] for the KTHNordic 32 system. Synthetic measurements used for off-line SE computations are obtained by running the models using the Power System Analysis Toolbox (PSAT) [13]. In the 9bus hybrid AC/DC test system a DC link between bus 7 and bus 8 replaces an $\mathrm{AC}$ line of the original $\mathrm{AC}$ system. Other parameters are unchanged. In the KTH-Nordic 32 hybrid $\mathrm{AC} / \mathrm{DC}$ test system a the DC link between bus 38 and 40 replaces the original AC line. More details on the KTH-Nordic 32 system can be found [15].

\section{A. Simulation results for the 9-bus system}

The state vector for the 9-bus AC system is organized as follows

$$
\mathbf{x}_{A C}=\left[\begin{array}{lllllllll}
\tilde{V}_{1} & \tilde{V}_{2} & \tilde{V}_{3} & \tilde{V}_{4} & \tilde{V}_{5} & \tilde{V}_{6} & \tilde{V}_{7} & \tilde{V}_{8} & \tilde{V}_{9}
\end{array}\right]^{T} .
$$

Synthetic measurements were obtained by performing time domain simulations in PSAT, where $0.02 \mathrm{~s}$ is selected as stepsize and a $0.1 \mathrm{pu}(10 \%)$ load increase is applied at bus 8 at $t=2$ s. Fig. 3 and Fig. 4 show the SE results for a single time instant (snapshot) and for multiple time instants. The SE residuals for the 9-bus AC system is lower than $10^{-13}$ p.u. (or deg).

$B$. Simulation results for the 9-bus hybrid AC/DC system

The state vector for the 9-bus hybrid AC/DC system is organized as follows

$$
\mathbf{x}_{A C / D C}=\left[\begin{array}{lllll}
\mathbf{x}_{A C}\left|\tilde{V}_{7}\right| \cos \alpha & \left|\tilde{V}_{8}\right| \cos \delta & V_{7 d c} & V_{8 d c} & I_{d c}
\end{array}\right]^{T} .
$$
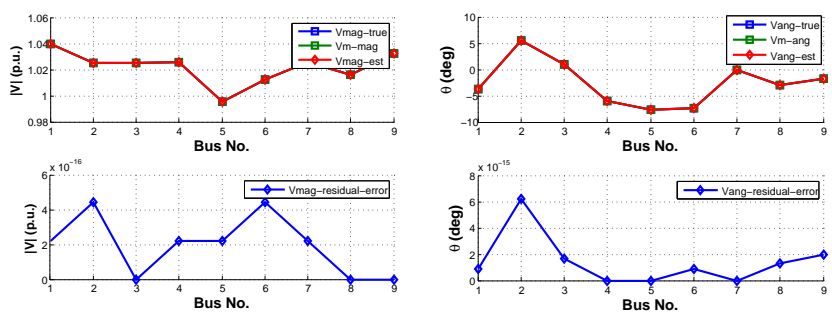

(a) Voltage magnitudes at all the (b) Voltage angles at one snapshot buses at one snapshot

(bus 7 is set as reference)

Fig. 3. LSE for the 9-bus AC system for a single snapshot

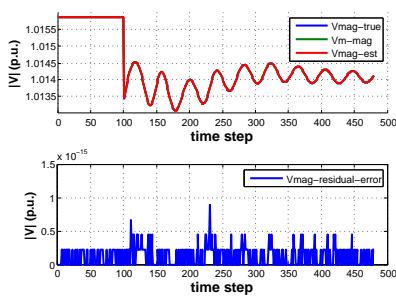

(a) Voltage magnitude at bus 8
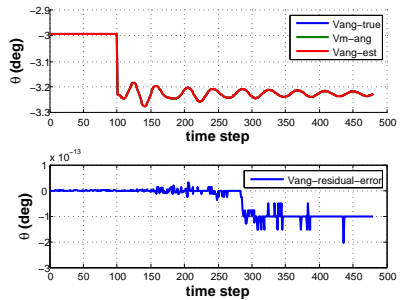

(b) Voltage angle at bus 8 (bus 7 is set as reference)
Fig. 4. LSE for the 9-bus AC system for multiple snapshots

The same test scenario as in Section IV-A is implemented. Fig. 5 and Fig. 6 show the computation results. In Fig. 5(b),

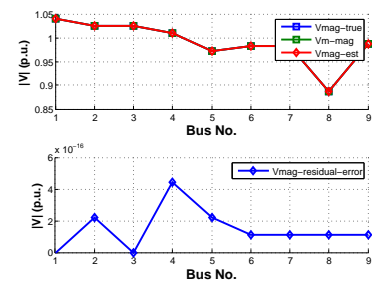

(a) Voltage magnitudes at all the buses at one snapshot

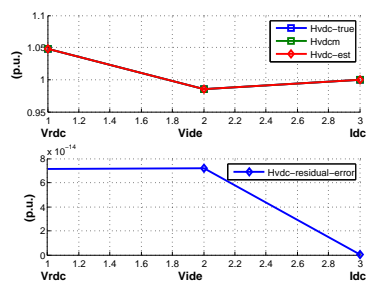

(b) DC state variables at one snapshot (before the perturbation)
Fig. 5. LSE for the 9-bus hybrid AC/DC system for a single snapshot

the DC states are computed before applying the perturbation, and estimated states have a small residual of $7 \times 10^{-14} p . u$. However, as shown in Fig. 6(b) 6(c) 6(d), when the system is subject to a perturbation, the SE residuals for DC states increase due to the limitation of static SE model. The static state estimator does not implement the dynamic DC link model and all the states are updated at the same rate of measurements, thus it is difficult to capture all the DC link dynamic response, especially when control schemes are active. This can also be confirmed by noting that the SE residuals decrease as the system oscillations decay. Regardless, the static SE residuals for DC states are still within acceptable values, except for the high peaks at the instant when the perturbation occurs. The hybrid $\mathrm{AC} / \mathrm{DC} \mathrm{SE}$ for $\mathrm{AC}$ states are as accurate as those in AC-only SE.

\section{Simulation results for the KTH-Nordic 32 system}

The state vector for the KTH-Nordic 32 system is organized as follows

$$
\mathbf{x}_{A C}=\left[\begin{array}{lllll}
\tilde{V}_{1} & \tilde{V}_{2} & \ldots & \tilde{V}_{51} & \tilde{V}_{52}
\end{array}\right]^{T} .
$$




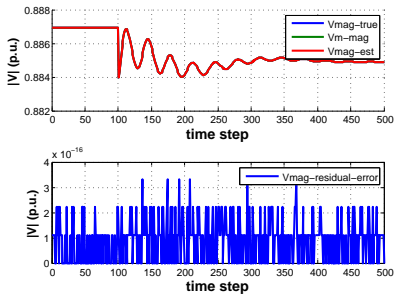

(a) Voltage magnitude at bus 8
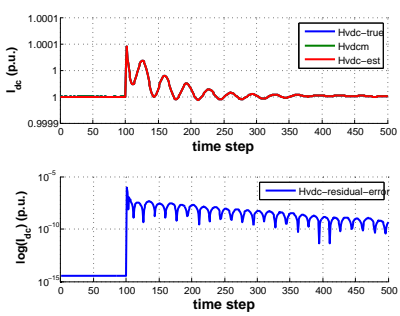

(c) DC current

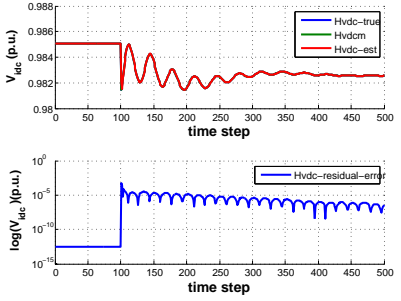

(b) DC voltage at bus 8

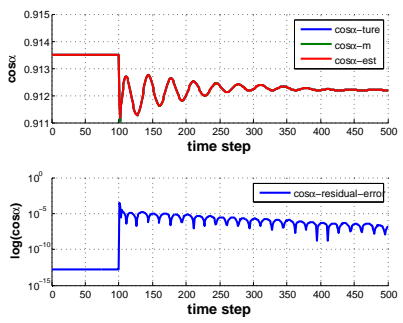

(d) $\cos \alpha$
Fig. 6. LSE for the 9-bus hybrid AC/DC system for multiple snapshots

Synthetic measurements are obtained with a step-size of 0.02 $\mathrm{s}$, and a $0.5 \mathrm{pu}(10 \%)$ load increase is applied at bus 40 at $t=2$ s. Fig. 7 and Fig. 8 show the SE computation results. The SE remain the same accuracy as that in the 9-bus AC system, which indicates that the proposed SE is not affected by the scale of system.
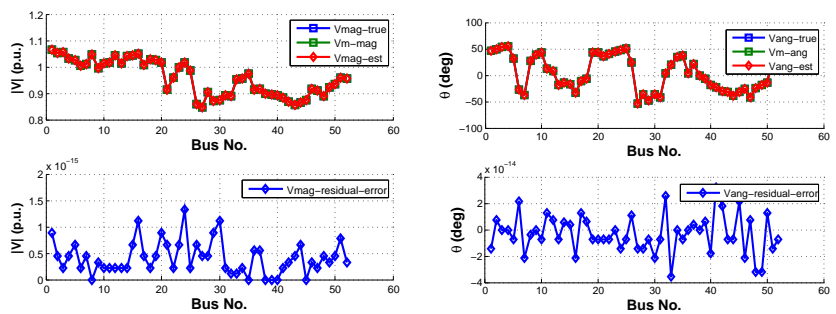

(a) Voltage magnitudes at all the (b) Voltage angles at one snapshot buses at one snapshot (bus 38 is set as reference)

Fig. 7. LSE for the KTH-Nordic 32 system for a single snapshot
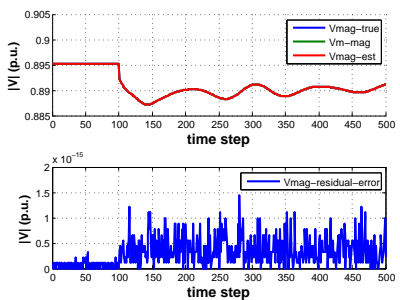

(a) Voltage magnitude at bus 40
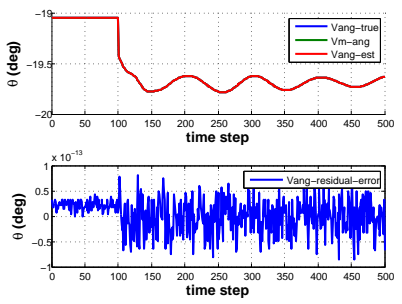

(b) Voltage angle at bus 40 (bus 38 is set as reference)
Fig. 8. LSE for the KTH-Nordic 32 system for multiple snapshots

\section{Simulation results for the KTH-Nordic 32 hybrid AC/DC system}

The state vector for the KTH-Nordic 32 hybrid AC/DC system is organized as follows

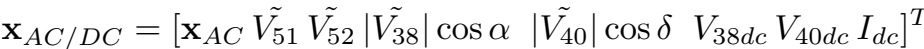

The same test scenario in Section IV-C is applied here. Fig. 9 and Fig. 10 show the SE computation results. Fig. 10(d) shows the true values of $\cos \alpha$ which is assumed constant (i.e. not measured). Observe that the estimated values have a small variation. This is due to the lack of DC link dynamics representation as explained in Section IV-B.
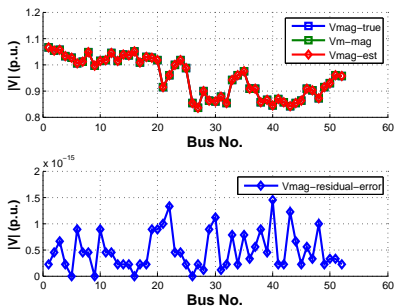

(a) Voltage magnitudes at all the (b) DC states at one snapshot (before buses at one snapshot

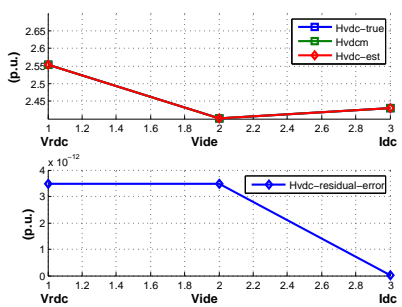

the perturbation)

Fig. 9. LSE for the KTH-Nordic 32 hybrid AC/DC system for a single snapshot
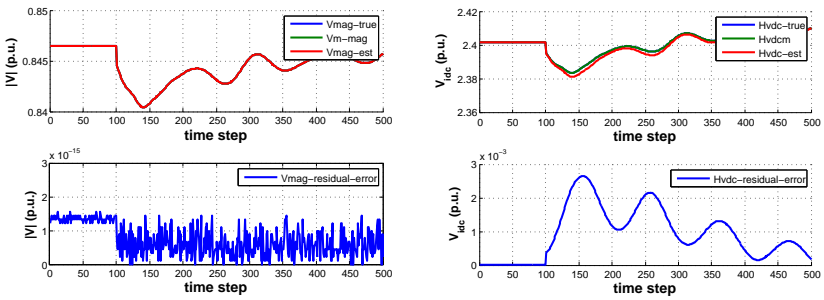

(a) Voltage magnitude at bus 40

(b) DC voltage at bus 40
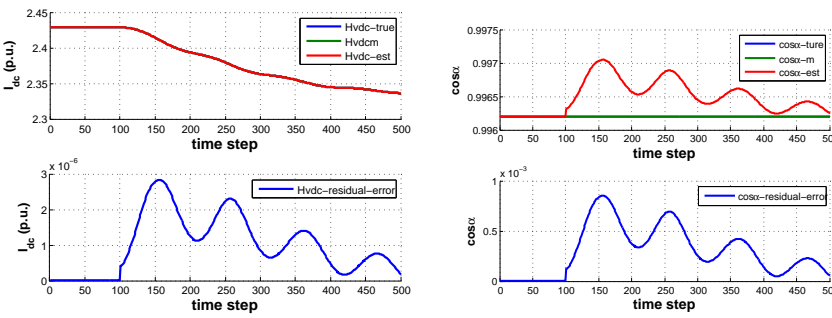

(c) DC current

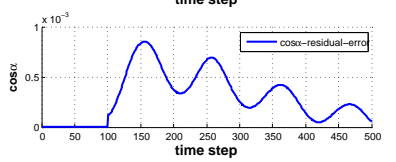

(d) $\cos \alpha$

Fig. 10. LSE for the KTH-Nordic 32 hybrid AC/DC system for multiple snapshots

\section{E. Effect of losing DC measurements}

1) AC/DC Measurement Redundancy Analysis: From (7) it can be shown that as long as two of five DC state measurements $\left(V_{r d c m}, V_{i d c m}, I_{d c m}, \cos \alpha_{m}\right.$ and $\left.\cos \delta_{m}\right)$ and the AC side voltage magnitudes $\left|\tilde{V}_{r}\right|$ and $\left|\tilde{V}_{i}\right|$ are provided, the three remaining DC states can be computed. In addition, normally $\cos \alpha$ and $\cos \delta$ remain relatively constant as in Sections IV-B and IV-D, thus $V_{r d c}, V_{i d c}$ and $I_{d c}$ can be computed assuming constant values for $\cos \alpha$ and $\cos \delta$. This brings a great advantage since $\mathrm{AC}$ measurements can be obtained using PMUs, and during normal operation only few DC measurements are needed.

When a DC measurement is lost, the corresponding rows in the residual matrix $(\mathbf{A})$, and the measurement matrix need to be removed. As long as the amount of rows is equal or larger than that of the number of columns in $\mathbf{A}$, all the states can be solved out by using the WLLS algorithm. The more rows . that $\mathbf{A}$ has, the higher the redundancy that the SE can offer. 
2) State Estimation with Reduced Number of DC Measurements: The same test scenario as in Section IV-B is applied here. Only $\cos \alpha_{m}$ and $\cos \delta_{m}$ are provided as DC measurements. As shown in Fig. 11, the residuals of the DC voltages and DC current increase while the residual of $\cos \alpha$ decreases. This is because $\cos \alpha$ is measured compared to previous case in Section IV-B. Nevertheless, observe that even with a limited number of DC measurements is is still possible to obtain acceptable state estimates for the whole AC/DC grid.

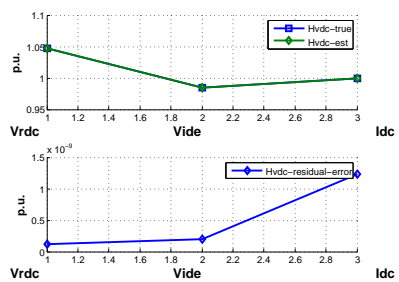

(a) DC state variables at one snapshot (before the perturbation)
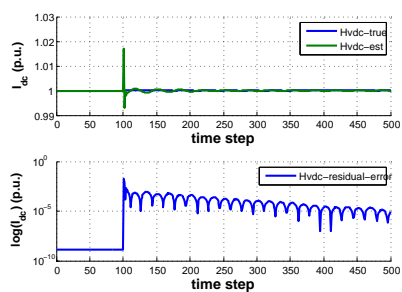

(c) DC current

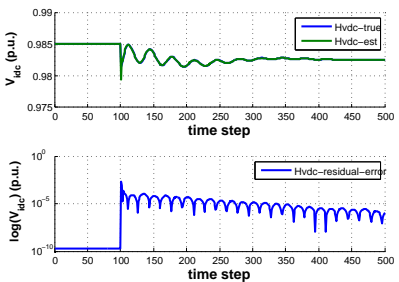

(b) DC voltage at bus 8
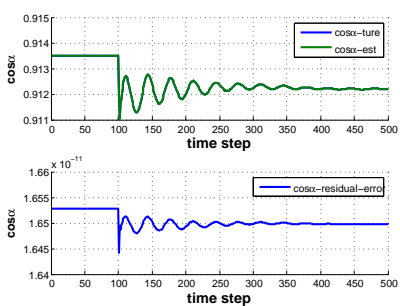

(d) $\cos \alpha$

Fig. 11. LSE for the 9-bus hybrid AC/DC system (without DC measurement redundancy)

\section{F. Effect of measurement noise}

Next, Gaussian white noise is added to the simulation results and considered in the SE process. The signal-to-noise ratio per sample is $75 \mathrm{~dB}$. The same test scenario of Section IV-C is applied on the KTH-Nordic 32 system. Fig. 12 shows the SE computation results, where the residual increases to $10^{-4}$ in comparison with $10^{-15}$ in the previous case. The state estimates are acceptable for the signal-to-noise ratio applied.
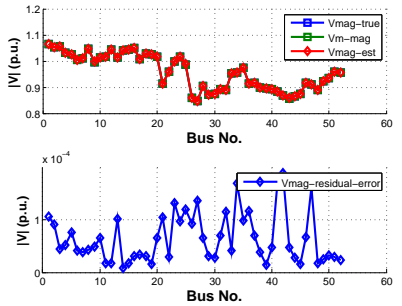

(a) Voltage magnitudes at all the (b) Voltage angles at all the buses at buses at one snapshot one snapshot

Fig. 12. LSE for the KTH-Nordic 32 system (with measurement noise)

\section{CONCLUSION AND FUTURE WORK}

A PMU-only state estimator for hybrid AC/DC systems has been introduced. By applying Kirchhoff's laws, the proposed network model simplifies the nonlinearities of the typical power flow network model used in conventional SEs. All the
AC and DC states are considered simultaneously to solve the LLS problem. After posing the network model and measurement model for this state estimator, simulation results for two power systems (both AC and hybrid AC/DC cases) are presented. In addition, it is feasible to estimate DC states with two of five DC measurements or even without DC measurements as long as $\cos \alpha$ and $\cos \delta$ are relatively constant. At last, the influence of measurement noise is discussed and it is shown that the SE algorithm can produce acceptable estimates under these conditions.

Future work will focus on measurement redundancy analysis, the inclusion of smoothing reactors in the classic HVDC link model, and the development of a VSC-HVDC link model including DC link capacitor dynamics. Both classic and VSCHVDC links will be included in the Phasor State Estimator (PSE) approach [5], [9] and contrasted with the algorithm presented in this paper.

\section{ACKNOWLEDGMENT}

The authors gratefully acknowledge the contributions of Prof. Joe H. Chow of the Rensselaer Polytechnic Institute in the form of valuable comments and suggestions to improve this paper.

\section{REFERENCES}

[1] A. Monticelli, "Real-Time Modeling of Power Networks" in State Estimation in Electric Power Systems-A Generalized Approach. Massachusetts: Kluwer Academic Publishers, 1999, ch.1, sec.3, pp.7-8.

[2] W. Sattinger, "Application of PMU measurements in Europe - TSO approach and experience," in Proc. IEEE Trondheim PowerTech 2011, 1 - 4 June, 2011.

[3] T.M. Haileselassie and K. Uhlen, "Power System Security in a Meshed North Sea HVDC Grid," Proceedings of the IEEE, vol. 101, no. 4, pp. 978-990, 2013.

[4] A. Meliopoulos, B. Fardanesh, and S. Zelingher, "Power system state estimation: Modeling error effects and impact on system operation," in Proceedings of 46th Hawaii Int. Conf. on System Sciences, Jan. 2011.

[5] L. Vanfretti, J. H. Chow, S. Sarawgi, B. Fardanesh, "A Phasor-Data-Based State Estimator Incorporating Phase Bias Correction,” IEEE Trans. Power Syst., vol.26, no.1, pp.233,119, Feb. 2011

[6] "IEEE Standard for Synchrophasor Measurements for Power Systems," IEEE Std C37.118.1-2011, Dec. 282011.

[7] Sirisena, H.R.; Brown, E. P M, "Inclusion of HVDC links in AC powersystem state estimation," Proc. IEEE, vol.128, no.3, pp.147,154, May 1981.

[8] Q. Ding, B. Zhang, T.S. Chung, "State estimation for power systems embedded with FACTS devices and MTDC systems by a sequential solution approach", Electric Power Systems Research, vol. 55, no. 3, pp.147-156, September 2000.

[9] S.G. Ghiocel, J.H. Chow, G. Stefopoulos, B. Fardanesh, D. Maragal, B. Blanchard, M. Razanousky, and D.B. Bertagnolli, "Phasor-MeasurementBased State Estimation for Synchrophasor Data Quality Improvement and Power Transfer Interface Monitoring," IEEE Transactions on Power Systems, available for early view. DOI: 10.1109/TPWRS.2013.2284098

[10] Phadke, A.G.; Thorp, J.S.; Karimi, K. J., "State Estimation with Phasor Measurements," IEEE Trans. Power Syst., vol.1, no.1, pp.233,238, Feb. 1986.

[11] A. Abur and A. G. Exposito, Power System State Estimation-Theory and Implementation. New York: Marcel Dekker, Inc., 2004, ch. 2, pp. 915.

[12] F. Milano, Power System Modeling and Scripting. London, UK: Springer-Verlag, 2010, pp.272-274.

[13] F. Milano, "An Open Source Power System Analysis Toolbox," IEEE Trans. Power Syst., vol.20, no.3, pp.1199,1206, Aug. 2005.

[14] F. Milano, "PSAT-Power System Anaylysis Toolbox Manual, 2.1.6 ed.," 2010, pp. 225-229, 404-406.

[15] Y. Chompoobutrgool, W. Li, and L. Vanfretti, "Development and implementation of a Nordic grid model for Power System small-signal and transient stability studies in a free and open source software," IEEE PES General Meeting, July 2012. 